\title{
Modeling the Variety of Trip Opportunities in Recreational Route Networks
}

\author{
András J. MOLNÁR \\ SZTAKI, Institute for Computer Science and Control, \\ Kende u. 13-17. H-1111 Budapest, Hungary; \\ Viator Association for Hiking and Culture, \\ Horánszky utca 20. H-1085 Budapest, Hungary; \\ E-mail:modras@sztaki.hu,molnar.andras.jozsef@gmail.com
}

\begin{abstract}
.
In this paper, we deal with the question of how the variety of trip opportunities can be modeled in - possibly complex - recreational trail networks (such as hiking paths or cycling ways). In order to quantify the variety of possible loop trips starting from specific trailheads (starting nodes accessible from outside the network) and the variety of connecting trips between specific origin-destination pairs, two novel measures of Loop Trip Variety Index (LTVI) and Connecting Trip Variety Index (CTVI) are proposed preliminarily and informally in [12], respectively, in the frame of assessing the impacts of some recent trail network developments. This paper establishes the formal definitions of improved variants of these measures, shows their well-definedness, presents the algorithms of their computation, investigates on their properties and benefits, and gives reasons of how and to what extent they can be treated as models of trip variety. Possible uses, application areas and future improvements are sketched especially for visitor management planning and profilebased trip recommendation systems.
\end{abstract}

Keywords. tourism and recreation, route network graphs, knowledge and information modeling, route planning, facility management, trip variety, network analysis

\section{Introduction and Related work}

Trails, such as hiking paths or cycling ways are used for outdoor activities and are available both for local recreation as well as reaching or exploring tourist destinations or being destinations themselves. They usually do not exist in isolation, as they form networks. Trip opportunities are determined by the network structure of specific modes of activity (hiking, biking, etc.) and the possible starting points (trailheads, such as parking places, bus stops, railway or ferry stations), trip destinations, and points of interest (POIs).

Current apps or systems provide either selected edited, prepared trips without any or minor variations, such as $[14,2,1,10]$, or a free navigation over the network, as in most mapping systems. If a user does not know the area or is not professional on maps, it might be difficult to find or plan a suitable trip route if the prepared, recommended trips (usually 
chosen with some subjectivity and with random overlaps) do not fit completely to one's profile. Based on these information, especially, planning a location for a longer holiday with multiple overnight stays can be difficult. Furthermore, if something changes in the network, its impact on trip opportunities of different target groups have to be assessed.

The field of recreational ecology has been emerged in the recent decades for studying the opportunities and impacts of field activities. The Recreational Opportunity Spectrum is one of the most utilized concept, which is based on local features and distances of specific types of features [6]. These works usually focus on local features and layout, environmental impacts at a global scale or along a longer trail, but not on assessment of structural properties of networks [16].

Works such as $[15,4,11]$ focus on conceptual modeling of mobility data and infrastructure. [9] analyses patterns in a network based on user trajectories. Prototypes of recommendation or assessment systems has been developed mainly for urban context but also for outdoor activities, some of them involving the social dimension $[13,17,19]$.

Graph theory has been extensively applied for transportation networks. Centrality measures such as node degree, closeness, betweenness centrality and others are effectively applied for them [8,5]. For outdoor trails, the situation is different. Trail usage differs from transport networks, and needs specific metrics to assess network plans and changes, and assist users to find attractive opportunities. It is a somewhat 'reversed situation' compared to transport networks. The purpose here is not to bring people from their particular original locations to (regular) desired destinations in the most effective way possible, and - in most cases - not to help users find the shortest way between two arbitrary locations, but to provide a pleasurable activity for a specified time frame, while considering the impact of these activities on the environment as well.

It has been revealed [3] that $60-64 \%$ of hikers prefer returning trips over linear hikes, and if we add the higher attractiveness of variation over repetition (at least for most users), minimizing necessary back-and-forth (dangling) sections and detours is reasonable by scoring circle trips and parts higher than those which need to be walked along the same way back. As different users have different aims and characteristics in their activities, developing a profile-based model seems to be beneficial.

Quantifying trip variations around a location, set of locations or between (sets of) locations can be trivially done by counting the number of possible trips in a length range. However, this number will show a combinatorial explosion, and because two different trips may have common sections, it will not be a useful metric to reflect on the actual variety of trip opportunities. If the network layout offers only trips without shared sections (such as a flower graph), counting these independent trips can be ideal. When, however, sections are shared, they must be somehow downgraded, and ideally, the measure of independent trips should be generalized, with looking at the maximal number of 'covering' trips of the reachable subgraph. Actual trip length should not make a difference if it falls into the range of the user's preference.

Modeling the variety of trip opportunities at nodes or sets of nodes of a trail network will provide a better understanding by an explicit expression of the knowledge related to possible trips contained by the network structure. It gives an instrument to help answering the following questions in particular:

1. For trail users with specific profiles: Where to go outdoors? Which is the variety of opportunities given by (a) specific trailhead(s) or origin-destination relations? Where can I find more variety if I consider staying somewhere for a couple of 
days? How many days shall I there to get a more or less full 'picture' of the area by a series of trips and how many repetitions of path sections I am likely to face?

2. For trail network managers: What is the impact of the existence or non-existence of a particular trail section (link) or set of sections in a network? Where and how shall we invest (to make new or upgraded trail sections) if we want to give more opportunities to trail users with specific profile(s)? In which locations shall we invest (trailheads, lodges) to improve facilities, transportation and their capacity if we want to focus on hubs with the best connectivity and variety (potential attractiveness for returning visitors)? How will the trip opportunities change or degrade across a larger area if we close or reroute a particular trail section?

Similar initiatives have recently begun in an urban context, such as [7], in which the effect of possible short additions to the bicycle network is investigated in terms of global city reachability. However, variety is not yet considered there.

The article [12] is proposing two novel metrics: the Loop Trip Variety Index (LTVI) and the Connecting Trip Variety Index (CTVI) along with two respective auxiliary measures of the Maximal Covering Loop Trips (MCLT) and the Maximal Covering Connecting Trips $(M C C T)$, in order to quantify the variety of possible loop trips starting from specific trailheads (starting nodes accessible from outside the network) and the variety of connecting trips between specific origin-destination pairs, respectively. These are proposed preliminarily and informally, in the frame of assessing the impacts of some recent trail developments in a real-life network scenario with some promising results.

This paper aims to contribute to the thorough discussion and formalization of these measures as models of trip variety, as proposed in [12]. We put them into a user-profilebased setting, and extend their applicability to sets of network nodes instead of single nodes. We add an index to reflect on back-and-forth sections (STVI), which were not considered at all, and include profile-based preferences such as the necessity of preferred POI visits in trips, which have substantial impact on the way of computation. A simple informal method of LTVI calculation is replaced by a full-fledged index computation algorithm, giving results of all 3 index components (CTVI,STVI,LTVI).

\section{Modeling User Profiles, Trail Networks and Trips}

Example 1 See Figure 1 taken from [11] with some modifications. Assume these are all walking paths. Line styles indicate difficulties, and the dotted lines are proposed extensions and improvements. Numeric labels denote section length in $\mathrm{kms}$. TNG denotes the graph without these additions, while ExtTNG includes the planned sections as well. ${ }^{1}$ Connected points of interests and trailheads are shown with pictograms, while capital letters denote nodes. We define two user profiles by personas, where both have no restrictions on the access mode or the format of their trips:

Adam or simply, Pa or a, who walks 5-20 km trips along both easy and difficult trails, has a preference for natural and landscape-related POIs (e.g. scenic spots).

Betty or simply $\mathrm{Pb}$ who walks shorter and easier trips, 3-10 km along easy sections only, but has no POI preference, likes walking for its own sake.

\footnotetext{
${ }^{1}$ The dotted line in parallel to section $Q K$ means the difficult section is to be upgraded as an easy section, so $Q K$ counts as an easy section in ExtTNG.
} 


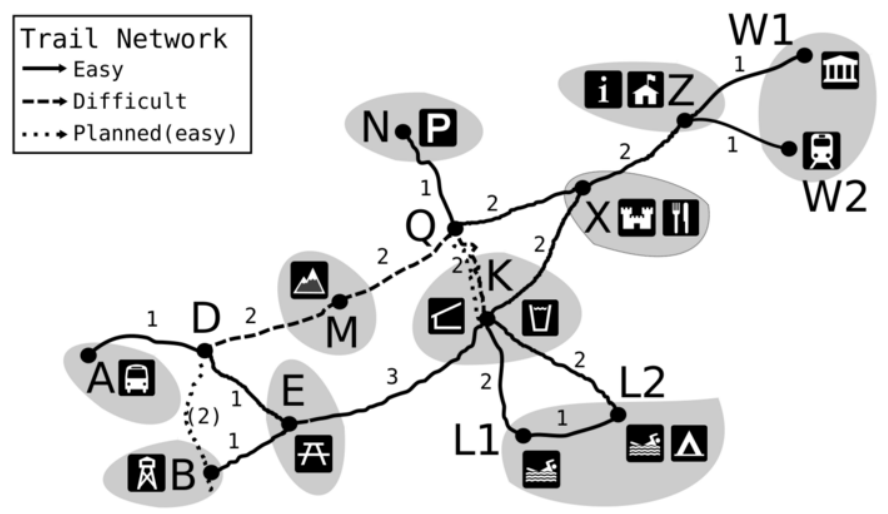

Figure 1. Network example with distances

Questions like the following may be asked for either profile, with our without the planned sections (how beneficial those will be for different profiles and cases):

- The variety of trips starting from a given node and returning there, such as $N$ (parking lot), K (lodge) or A (bus stop).

- The variety of trips connecting two specific nodes or sets of nodes, such as $A \rightarrow W 2$ (public transport), or $W=W 1, W 2 \rightarrow L=L 1, L 2$ (city to lake \& camp).

We characterize the needs and preferences of trail users by the followingfields:

Activity mode Hiking or biking, or any other modality of activity exercised along the trails. A finite set ActModes is assumed to be given with the possible values.

Trail and trip type/difficulty What type of trail(s) is the user ready to go along a trip, in accordance to one's equipment and preparedness (technical difficulty level). We assume the finite set of possible values (which may be a direct product of multiple relevant trail section parameters) are given by TrailTypes $_{\text {act }}$ for each act $\epsilon$ ActModes. Furthermore, the set TripTypes act $_{\text {for }}$ each act $\in$ ActModes proposes a set of typical trip types defined by a function allowedTrailTypes act to allowed trail types, such that $\forall$ tripType $\in$ TripTypes $_{\text {act }}:$ allowedTrailTypes act $_{\text {tripType }} \in$ $2^{\text {TrailTypes }}$ act . The universal trip type set is TripTypes $=\bigcup_{a \in \text { ActModes }}$ TripTypes $_{a}$.

Trip length range the user is ready to take (min, max), in $\mathrm{km}$. We assume a finite set of

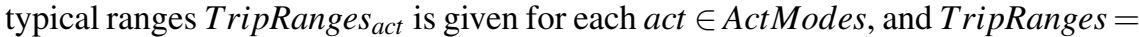
$\bigcup_{a \in \text { ActModes }}$ TripRanges $a$. The actual min and max distances of range are available using functions min, max : TripRanges $a c t \rightarrow \mathbb{R}^{+} .^{2}$

Returning trips only A boolean value determining whether the user wants to take trips only which return to their starting point, or ending possibly somewhere else.

Access mode How the user gets to the trailhead (the starting point of the trip). Mainly car or public transport. Possible values are given as the finite set AccModes, including

\footnotetext{
${ }^{2}$ In practice, the distance is usually enhanced by the ascent and descent in meters by section and forms a basis for calculating the ideal time for completing the trip. We push the elevation and time issues under the trail/trip type/difficulty part and keep the distance range in order to keep simplicity here; which is, on the other hand, independent of the direction of moving along a trail.
} 
a special value $T$ meaning no access mode is given and the trip can start and end in any node of the network.

POI preference A finite set of types of points of interest relevant for the user and wanted to be included in one's trip. Types are defined by PoiTypes, and a subset is given for each profile, meaning the user wants to undertake such trips only which include visiting of at least one point of interest whose type is in the set (it can be the starting or ending point of the trip as well). Detours are taken only to such types of POIs. The empty set means no preference, i.e. no restriction on the user trips.

Definition 1 The characteristics of trips a user is willing to take is defined by the Trail User Profile: a tuple $p=($ actMod,tripType,tripRange, retOnly,accMod,poiTypes $)$ over ActModes $\times$ TripTypes $\times$ TripRanges $\times\{\perp, \top\} \times$ AccModes $\times 2^{\text {PoiTypes }}$ where tripRange $\in$ TripRanges actMod $_{\text {and }}$ tripType $\in$ TripTypes actMod.

Example 2 The user profiles of Example 1, given the single activity walk and trip types by allowedTrailTypes $=\{$ easyTrip $\mapsto\{$ Easy $\}$, anyTrip $\mapsto\{$ Easy,Difficult $\}\}$ are:

- $P a=($ walk, anyTrip $,(5,20), \perp, \top,\{$ mountain, spring, lake, lookout, infocentre $\})$

- $P b=($ walk, easyTrip $,(3,10), \perp, \top, \emptyset)$

A single user may have multiple profiles being active depending on the actual situation (e.g. time availability).

We assume the trail network is given in form of an undirected graph (assuming most trails are bidirectional, adaptation to directed sections is a future issue), a classical geospatial routable graph structure enhanced by specific labels corresponding the user profiles in the following way.

Definition 2 A Trail Network Graph is a labeled graph $T N G=\left(T N, T S^{+}\right.$, link $\left.^{+}, p n, p s\right)$ where $T N=$ tnodes $(T N G)$ is the finite set of trail nodes, $T S^{+}=$tsections $^{+}(T N G)$ is the finite set of (directed) trail sections, link ${ }^{+}: T S^{+} \rightarrow T N \times T N$ is a function defining the linkage of nodes by the sections. Furthermore, $p n=($ geoCoords, accModes, poiTypes $)$ and $p s=($ geoPath, length, actModes, trailType $)$ are tuples of functions defining properties of nodes and sections, respectively.

The sections are considered to be bidirectional (undirected graph) with each section represented in a specified direction in $\mathrm{TS}^{+}$. Therefore, we assume a natural extension of the above notations to a symmetric setting where each section and its reversal is present with a reversed linkage extension. We omit the ${ }^{+}$upper indices for this extension.

The function rev over TS defines the reversal of each section. The link function can be specified as a tuple of functions (start, end). With these notations, we can denote a TNG with a tuple (TN,TS, (start, end), rev, pn,ps) as well.

Properties ps of each trail node n: geoCoords $(n)$ is a pair of geospatial coordinates (latitude-longitude), accModes $(n) \subseteq$ AccModes $\backslash\{\top\}$ defines the access modes for trailhead nodes (possible starting point of trips), poiTypes $(n) \subseteq$ PoiTypes defines the types of points of interests located at $n$.

Properties ps of each trail section $s$ : geoPath $(s)$ is the linestring geometry, length $(s) \in \mathbb{R}^{+}$is the length in $\mathrm{km}$, actModes $(s) \subseteq$ ActModes defines the possible ac-

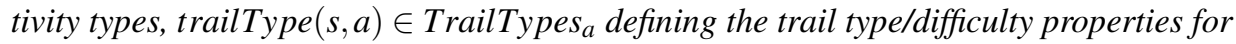


each $a \in \operatorname{act} \operatorname{Modes}(s){ }^{3}$ For topological consistency, we require the coordinates of start and end points of sections match the nodes they connect.

Furthermore, we assume the nodes and sections are uniquely identified by their geographical coordinates, so one can 'point out' a node or segment by its coordinate position(s). Besides that, a name and/or a technical identifier can be given but it is not relevant for our discussion.

For simplicity, we use the nodes in our examples to identify the trail sections, as we do not have parallel edges in our network graph.

Given a set of activity and/or trail types, a trail network graph can be restricted by filtering its sections valid for the given activity and trail types, respectively.Given a set of access modes and/or poi types, the respective nodes of a trail network graph can be selected. The Mode-specific Trailheads for an access mode accm are denoted by Trailheads $(T N G, a c c)$. The Locations of POIs (or simply, POIs) are denoted by $\operatorname{Poi}(T N G, p t s)=\{n \in \operatorname{tnodes}(T N G) \mid \operatorname{poiTypes}(n) \cap p t s \neq \emptyset\}$ and, in general, $\operatorname{Poi}(T N G)=\{n \in \operatorname{tnodes}(T N G) \mid \operatorname{poiTypes}(n) \neq \emptyset\}$.

Based on the trail network graph-related definitions, we give definitions of trips, which users can undertake in a trail network graph:

Definition 3 A trip $t$ in a trail network graph $T N G$ is a sequence $\left(s_{1}, s_{2}, \ldots, s_{n}\right)$ of connected (directed) trail sections of $T N G$ where start $\left(s_{i+1}\right)=$ end $\left(s_{i}\right)$ for each $i \in$ $\{1, \ldots, n-1\}$.

- The length of the trip is the sum of lengths of its sections along the sequence.

- The starting and ending nodes of the trip are defined as $\operatorname{start}(t)=\operatorname{start}\left(s_{1}\right)$, end $(t)=$ end $\left(s_{n}\right)$, respectively.

- The set of all possible trips of TNG is denoted by Trips $(T N G)$. Since repetitions in trips are allowed, this is not a finite set if the graph contains any sections.

- The subgraph of TNG defined by the nodes and sections of a set of trips TripSet is denoted by TripG(TNG, TripSet).

- Consistently with referring trail sections by their start and end nodes (see above) we use a sequence of trail nodes to describe trips if no parallel edges are present.

- We define specific types of trips or parts of trips in the following.

\section{Definition 4}

- Two (or more) trips are called independent trips if they have no sections (pairwise) in common, and no reversal of a section occurs in (any of) the other trip(s).

- A section or sequence of adjacent sections in a trip is called a repeated part if it occurus at least twice in the same trip (in the same direction).

- A simple tripis a trip without any repeated sections. Where not stated explicitly otherwise, we are considering simple trips only from now on.

- A back-and-forth part of a trip is a section (or sequence of adjacent sections) whose reversal occurs along the same trip (not necessarily directly following it).

- A dangling part in a trip, a.k.a. spike, is a sequence of sections in a trip where the hiker walks back the same way in reversed direction immediately. The turning point of a dangling part (spike) is its middle node.

\footnotetext{
${ }^{3}$ Properties $p s$ of a reversed trail section are equal to the properties of the original section except geoPath $(\operatorname{rev}(s))$ which is the reversed sequence of geoPath $(s)$.
} 
- A circle is a single section or a connected series of sections along a trip (not necessarily following directly one another) if it returns to its starting point at its end and no node or section (or its reversal) is repeated along it.

- A loop is a single section or a series of subsequent sections in a trip, or a full trip, if it returns to its starting point at its end and contains at least one circle. ${ }^{4}$ A loop may contain circles and back-and-forth sections (even including spikes as well).

- Two specific types of loops are identified: an 8-shaped-loop has a circle in it, which being removed from the loop results solely another circle. A P-shaped-loop is a loop having a circle in it, which being removed from the loop results a spike leading originally to the circle.

- A trip is a returning tripif start $(t)=e n d(t)=n$. A returning trip is either a loop trip or a non-loop returning trip, containing only back-and-forth sections.

- A non-returning trip is called a connecting trip.

- A direct trip is a connecting trip not containing any loops or spikes in it.

- A visiting trip in reference of a specific set of preferred nodes $V N \subseteq T N$ denoted by $V \operatorname{Trip}(t, V N)$ is a trip having at least one node being a member of $V N$. It can be the starting or ending point of the trip as well.

- Similarly, a strict visiting trip, denoted by $\operatorname{StVTrip}(t, V N)$, is a visiting trip in which a dangling section is allowed only if its turning point is a member of $V N$.

- Given StartTN,DestTN,VisTN $\subseteq T N$, the set of possible trips between StartTN and DestTN is TripsFromTo(TNG,StartTN,DestTN), is the set of trips $t$ in $T N G$ for which start $(t) \in \operatorname{Start} T N$, end $(t) \in$ EndTN (not necessarily simple). STripsFromTo(TNG,StartTN,DestTN) is defined in a similar way, denoting simple trips only. In order to shorten the notations, if any of the sets StartTN,DestTN is a singleton, it can directly be replaced by its member; and if it equals $T N$ (thus meaning no restriction) it can be replaced with the marker $T$.

- Given $\min L, \max L \in \mathbb{R}^{+} \cup\{\infty\}$, the set of possible trips of length range between $\min L$ and maxL is denoted by LRangeTrips $(T N G,(\min L, \max L)=\{t \in$ $\operatorname{Trips}(T N G) \mid \min L \leq$ length $(t) \leq \max L\}$. SLRangeTrips $(T N G, \min L, \max L)$ is defined in a similar way, denoting simple trips only.

- Given a network (sub)graph TNG. A set of trips TSet is a cover (or covering trip set) of TNG if each section s of TNG appears in at least one of the trips in TSet, and each trip in TSet has at least one section not occurring in all other trips of the set. A maximal cover is a cover with the maximal number of trips. This concept can be refined by a predicate $P$ as a trip property where only trips of that property are allowed and sought for the (maximal) cover.

Example 3 With the above concepts:

- Trailheads of our network in Figure 1 are $\{A, N, W 2\}$ (access points to transport), however, trips are allowed to start at other nodes as well, for instance, at nodes $K$, L2 (lodge or campsite) or $W 1$ (city residential area).

- The trip $t 1=(N, Q, X, K)$ is a simple direct connecting trip (although not the shortest one), while $2=(N, Q, X, Z, X, K)$ is a simple connecting trip which is not direct. $t 2$ has a back-and-forth part (a spike) (X,Z,X) and has no loops, is a strict visiting trip w.r.t. POI type infocentre, and a visiting trip (but not strictly) w.r.t.

\footnotetext{
${ }^{4}$ Note that loops usually defined as single reflexive links in graphs. We use the term loop in a wider meaning.
} 
parkinglot, castle, restaurant, lodge, spring. $t 1$ is a strict visiting trip for these $P O I$ types and is not a visiting trip for type in focentre.

- The trip $t 3=(K, Q, X, Z, X, K, L 1, L 2, K)$ is a simple returning trip with circle parts $(K, Q, X, K)$ and $(K, L 1, L 2)$. The latter is a spike-free loop part of it, another loop part is $(K, Q, X, Z, X, K)$, they form a 8-shaped loop together, and the whole trip can be called as a loop trip. It is independent of the trip $t 4=(K, E, B, E, K)$, which is a non-loop returning trip (it is a spike). If we add the planned section $B, D$ to the network, the trip $t 5=(K, E, B, D, E, K)$ becomes available as a $P$-shaped loop trip. All loops are simple loops.

Whether a trip corresponds to a (/set of) user profile, is defined in the natural way:

Definition 5 Trip-profile matching: Given a trail user profile $p=\left(\right.$ actMod $_{p}$, tripType $_{p}$, tripRange $_{p}$, retOnly $_{p}$, accMod $_{p}$, poiTypes $\left._{p}\right)$ and a trail network graph $T N G=(T N, T S,($ start, end $), r e v,($ geoCoords, accModes, poiTypes $)$, (geoPath, length, actModes, trailType)) and a trip $t$ in $T N G$, we say $t$ matches (is acceptable for) $p$, denoted by MatchTP $(t, p)$, iff

- its sections correspond to the activity mode(s), trail and trip type(s)/difficulty(/ies)

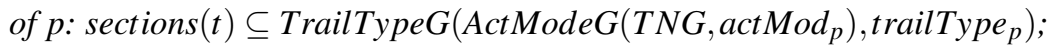

- its length falls between the trip length range of $p$ : $t \in$ LRangeTrips $\left(T N G, \min \left(\right.\right.$ tripRange $\left._{p}\right), \max \left(\right.$ tripRange $\left.\left._{p}\right)\right)$;

- it is a returning trip if $p$ requires that: retOnly $\rightarrow \operatorname{start}(t)=e n d(t)$;

- its starting and ending point have the access mode of $p$ : accMod $\operatorname{ad}_{p} \neq \top \rightarrow t \in$

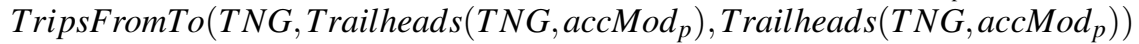

- it is a strict visiting trip for preferred POI types: poiTypes $_{p} \neq \emptyset \rightarrow \operatorname{StVTrip}\left(\operatorname{Poi}\left(T N G\right.\right.$, poiTypes $\left.\left._{p}\right)\right)$.

For a set of trail user profiles $P$, $t$ corresponds to $P$, denoted by $\operatorname{MatchT} P(t, P)$, iffor any $p \in P$ t corresponds to $p$.

A relaxation (fuzzification) of the definition is given in order to allow shorter simple trips than requested - these offer something but not fully match -, with a match degree:

Definition 6 Given a trail user profile $p$ and a trip $t$ as above, we define the match degree of t for $p$, denoted by MatchT PD $(t, p)$ as being 1 if MatchTP $(t, p)$; otherwise, the value $\frac{\text { length }(t)}{\text { min(tripRange })}$ if $t \in$ LRangeTrips $\left(T N G, 0, \max \left(\right.\right.$ tripRange $\left.\left._{p}\right)\right)$ and obeys the other, non-length-range-relevant conditions of matching as defined above; and 0 for all others.

We can define the 'personalized' (profile-relevant) subgraph of a trail network graph for each (set of) trail user profile(s), which contains nodes and sections only reachable by a user with the given profile(s), and optionally a set of acceptable trip starting and destination nodes.

Definition 7 Assume a set of trail user profiles $P$, a trail network graph TNG with nodes $T N$ and sections $T S$ are given with a set of acceptable starting nodes StartTN $\in$ TN (trailhead locations), the set of acceptable destination nodes Dest TN $\subseteq T N$.

The P-matching (simple) trips of TNG w.r.t. (StartTN,DestTN), denoted by ProfileMatchST (TNG,P,StartTN,DestTN), consists of all possible simple trips $t$ in $T N P \subseteq T N$ for which MatchTP $(t, p) \wedge \operatorname{start}(t) \in \operatorname{Start} T N \wedge$ end $(t) \in \operatorname{Dest} T N$ is true. 
The P-relevant (simple) trips of TNG w.r.t. (StartTN,DestTN), denoted by ProfileRelevST (TNG,P,StartTN,DestTN), consists of all possible simple trips $t$ in $T N P \subseteq T N$ for which MatchTPD $(t, p)>0 \wedge \operatorname{start}(t) \in \operatorname{Start} T N \wedge$ end $(t) \in \operatorname{Dest} T N$.

The P-relevant trail network subgraph of TNG w.r.t. (StartTN,DestTN), denoted by ProfileRelevG(TNG,P,StartTN,DestTN), consists of nodes $T N P \subseteq T N$ and sections TSP $\subseteq$ TS being part of any $t \in$ ProfileRelevST(TNG, P, StartTN,Dest TN).

Example 4 Recall the user profiles and network from Examples 1, 2.

- Examples of Pb-relevant trips w.r.t. $(L 2, L 2)$ (returning) are:

$(L 2, K, X, K, L 1, L 2),(L 2, L 1, L 2)$. The latter is not a match, as it is shorter than specified $(3 \mathrm{~km})$, but will count with relevance score $2 / 3$.

- The Pb-relevant subgraph between nodes $N, W 2$ is covered by the two matching trips $(N, Q, X, Z, W 1, Z, W 2),(N, Q, X, K, X, Z, W 2)$.

\section{Trip Variety Indices}

The following subsections define the core indices of our contribution. The main motivation is to quantify the length proportion of not yet visited sections of different types (connecting, loop/circle, dangling/spike) in a sequence of trips and look at/between certain locations how high this number can be if a user visits all reachable sections combined into trips matching one's profile.

Definition 8 Assume a (finite) series of arbitrary, subsequent trips SeqT a user is taking one after another.

- The trip novelty ratio of the $i^{\text {th }}$ trip $t_{i}$ in SeqT is the ratio of summed section lengths of $t_{i}$ not yet visited before (not being part of any $t_{j}, j<i$ ), divided by the total length of $t_{i}$.

- The summed novelty ratio of SeqT is the sum of novelty ratios for all $t_{i}$ in SeqT.

When a location or multiple locations (nodes) are given with a user profile, we consider all possible trips from that location and try to maximize these values. That means, in a maximized case, we assume a user takes trips in a sequence with the minimum possible change than the previous one, and accumulate the length proportions of the newly visited sections(s).

We, however, give priority to (direct) connecting trips first, then remaining loops and then, the rest is are those dangling sections, which can only be visited in a back-andforth manner (spike in a trip); this way, we can differentiate the trip variety of such trip formats and index them separately. Such ordering is called a $\mathbf{C}-\mathbf{L}$ prioritized ordering of possible (P-relevant) trips. ${ }^{5}$

\footnotetext{
${ }^{5}$ Different approaches could be resulted by evaluating the variety of connecting trips with/without loops and/or spikes, returning loop trips with/without spikes, and returning trips with spikes independently of each other. However, we wanted to construct a composite index whose components are additive and give a single value of (prioritized) variety under flexible circumstances, as we consider the start and destination nodes as sets of nodes and whether connecting or returning trips are allowed, is determined by their intersection and (symmetric) set difference.
} 
It is possible that a user can explore the reachable part of the network in less number of trips fitting to one's profile. If the variety index equals the number of trips maximized case above, it means all possible trips (or remaining parts of the respective format - such as loop or spike) are independent. A reversal of a trip is considered as the same, adding no more variety. If we considered non-simple trips as well, it is obvious that their novelty ratios will not be higher than simple trips.

Examples and more discussion will be given after the definitions.

Definition 9 Let $T$ Set be a set of simple trips in a trail network graph TNG.

For each section s in TripG(TSet), we specify a set of trips in order to determine the characteristic role of $s$ in the given set of trips, and then assign a weight to s based on that.

- Let DirectTrips (s,TSet) (Direct trips of $s$ ) be the set of trips in TSet in which $s$ is not a member of a loop (circle or back-and-forth section).

- Let CLoopTrips(s,TSet) (Circle-Loop Trips of $s$ ) be the set of trips in TSet in which $s$ is a member of a circle.

- Let BFTrips(s,TSet) (Back-and-Forth Trips of $s$ ) be the set of trips in TSet in which $s$ is a member of a back-and-forth section.

When defining the variety indices, one may give a priori weights to the trips being evaluated. A function tw: TSet $\rightarrow \mathbb{R}_{0}^{+}$is used for this. By default, it is constant 1 and in this case, it can be omitted from the notations.

The weights and indices:

- The General Trip Variety Index of TSet given their a priori weights tw, denoted by GTVI (TSet, tw) is a weighted sum of all sections in TripG(TSet), where the index weight of each network section $s$, denoted by $w_{G T V I}(s, T$ Set,$t w)$ is determined by the ratio of its length and the weighted length of the shortest trip with the highest a priori tw value of TSet containing $s: w_{G T V I}(s, T$ Set,$t w)=\frac{\text { length }(s)}{\operatorname{tw}(t) * \operatorname{length}(t)}$ where $s \in t s(t), t \in \operatorname{argmax}_{t w}(T)$ with $T=\operatorname{argmin}_{\text {length }}(T S e t) .{ }^{6}$ The notation is extended to sections not in TSet by assigning a weight of 0 . We omit tw if it is constant 1 : GTVI $($ T Set, 1$)=G T V I($ TSet $)$.

- The (General) Connecting Trip Variety Index, denoted by GCTVI(TSet, tw) is a similar weighted sum over each section, where the weight $w_{G C T V I}(s, T$ Set,$t w)=$ $w_{G T W I}(s$, DirectTrips $(s, T$ Set $), t w)$. It is also called simply CTVI, and $w_{C T V I}$.

- The (General) Loop Trip Variety Index, denoted by GLTVI(TSet,tw) is a similar weighted sum over each section, where the weight $w_{G L T V I}(s, T S e t, t w)=$ $w_{G T W I}(s, C L o o p T r i p s(s, T S e t), t w) .{ }^{7}$ A strict variant of GLTVI is the simply called LTVI, which acts as a supplement to CTVI, where only the non-connecting sections are counted here: $w_{L T V I}(s, T$ Set,$t w)=0$ if $w_{G C T W I}(s, T$ Set,$t w) \neq 0$, otherwise it equals $w_{G L T V I}$.

- The (General) Spike Trip Variety Index, denoted by GSTVI(TSet,tw) is a similar weighted sum over each section, where the weight $w_{G S T V I}(s, T S e t, t w)=$

\footnotetext{
${ }^{6}$ The functions argmin and argmax are assumed to return subsets because multiple items may have the same $\max / \mathrm{min}$ value.

${ }^{7}$ The term loop refers to the phenomenon that sections of circles are parts of loop trips or loop parts of connecting trips. We quantify the variety of circles of possible loops here.
} 
$w_{G T W I}(s, B F T r i p s(s, T S e t), t w){ }^{8}$ A strict variant of GSTVI is the simply called STVI, which acts as a supplement to CTVI and LTVI, where only the nonconnecting and non-circle sections are counted here: $w_{S T V I}(s, T$ Set,$t w)=0$ if $w_{G C T W I}(s, T$ Set,$t w)+w_{G L T W I}(s, T$ Set,$t w) \neq 0$, otherwise it equals $w_{G S T V I}$.

- The Composite Trip Variety Index is denoted and defined by the triplet CompTVI $(T S e t, t w)=(C T V I(T S e t, t w), L T V I(T S e t, t w), S T V I(T S e t, t w)) . \quad I t$ reveals more details of the structure of trips in TSet than the GTVI.

- If all trips start and end at the same node, CTVI (T set, $t w)=0$ and the Composite Returning Trip Variety Index can also be used instead: CompRTVI(TSet,tw) = (LTVI(TSet,$t w), S T V I(T$ Set,$t w))$.

- The Summed C-L prioritized Trip Variety Index is defined by $\operatorname{SumTVI}(T S e t, t w)=C T V I(T S e t, t w)+\operatorname{LTVI}(T S e t, t w)+\operatorname{STVI}(T S e t, t w))$.

Note that each section is calculated only once in each index, and in only one of the components of CompTVI, regardless of how many trips the section is contained by.

The main step in the definition follows. We intend to give variants of the above variety indices based on visitor profiles and locations in the network where the index represents all possible trips given that context.

We do not allow longer trips than requested by the profile, but shorter ones are allowed partially, as their length is divided by the minimum allowed length. This approach not only gives an indication of partial relevance to a trail user (and reflects the option for the user to repeat a shorter trip as a non-simple trip to reach the min range length value), but will also help in the effective computation.

Definition 10 Let TNG be a trail network graph with nodes TN, P a trail user profile, Start $T N \in T N$ (possible starting locations) and the set of acceptable destination nodes Dest $T N \subseteq T N$.

The Profile Based (Composite) Trip Variety Index is denoted and defined by the triplet CompTVI $(T N G, P$, Start TN, DestTN $)=(C T V I(T N G, P, \operatorname{StartTN}, \operatorname{Dest} T N)$, LTVI $(T N G, P, \operatorname{StartTN}, \operatorname{Dest} T N), \operatorname{STVI}(T N G, P, \operatorname{StartTN}, \operatorname{Dest} T N))$ where

- $\operatorname{CTVI}(T N G, P, \operatorname{StartTN}, \operatorname{Dest} T N)=$ CTVI(ProfileRelevST (TNG,P, StartTN,DestTN), length/min(tripRange $P))$ is the connecting trip variety index,

- $\operatorname{LTVI}(T N G, P, \operatorname{Start} T N, \operatorname{Dest} T N)=$ LTVI(ProfileRelevST (TNG,P,StartTN,DestTN),length/min(tripRange $P))$, is the loop trip variety index,

- $\operatorname{STVI}(T N G, P, \operatorname{Start} T N, \operatorname{Dest} T N)=$ STVI(ProfileRelevST (TNG, P,StartTN,DestTN), length/min(tripRange $)$ ), is the spike trip variety index.

Usually, the index is applied to singleton sets where specific nodes (mostly trailheads) are evaluated by the variety of possible trips the network offers. In this case, Start $T N=\{$ start $\}$, Dest $T N=\{$ end $\}$, and the members can be written directly instead of the sets: CompTVI(TNG, P, start, end $)$, etc.

\footnotetext{
${ }^{8}$ The term spike refers to the phenomenon that if circles are omitted from loop trips or sections, the remaining sequence becomes a composition of spikes (dangling sections). This index includes values for all sections in back-and-forth parts of loop trips even if they do not form a spike in strict manner in their trip originals.
} 
If StartTN $=$ Dest TN $=\{t n\}$ then we may omit the first item and call it the Composite Returning Trip Variety Index instead:

CompRTVI $(T N G, P, t n)=(\operatorname{LTVI}(T N G, P, t n), \operatorname{STVI}(T N G, P, t n))$.

Proposition 1 The Profile Based (Composite) Trip Variety Index (and all the above defined indices) are well defined.

Well-definedness is ensured by, first of all, the well-definedness of GTVI, since if a section $s$ is in multiple trips, their minimum length is unique (lengths are positive and we consider only simple trips), and even if there are multiple min-length trips of a specific format (connecting, returning with/without loops) containing $s$, the weight depends only on their, unique, length value, and the a priori trip weight which is maximized among them. An actual choice of the next shortest trip (if there are multiple available) will have no influence on the later assigned weight values of sections of the other shortest-tripoptions, since trips with a given (min) length are exhausted before any of the longer trips are considered for section weighting. Furthermore, CompTVI for a set of trips explicitly assigns a nonzero weight to $s$ only at most one of its component indices. The allowed ordering of specific formats (direct connecting, remaining loop, remaining spike) ensures each section will be assigned to a specific component index with a nonzero value, regardless of the actual ordering of the trips in the sequence, as each section being part of any allowed direct connecting trip will be exhausted before any other loops are considered, and all possible circle sections are exhausted before the remaining possible spike sections are scored with weights. For given parameters, ProfileMatchST and ProfileMatchG are finite, and each section $s \in$ ProfileMatch $G$ has at most one component index in Pbased CompTVI with a nonzero value, and it is not dependent on the order of trips which component index is nonzero for a specific section.

In order to provide an even better insight on the variety, namely, to see the scale of the above indices, the number of trips covering the opportunity space is useful to add.

Definition 11 With the above notations,

a set of trips TCov, w.r.t. (TNG,P, StartTN,DestTN) is a

- Covering Connecting Trip set (CCT) if it contains connecting trips of ProfileRelevST(TNG,P,StartTN,DestTN)), covering the direct connecting trips between (StartTN,DestTN) in

TripG(ProfileRelevST (TNG, P, StartTN,DestTN)), where each trip has at least one section not occurring in all others, and each trip has a minimum possible length with these conditions. ${ }^{9}$ A Maximal CCT (MCCT) is a CCT with the highest number of trips. The number MCCT (TNG,P,StartTN,DestTN) is the weighted count of trips in an MCCT, each multiplied by their MatchTPD value.

- Covering Loop-containing Trip set (CLT), if it contains (non-direct) connecting trips or or loop trips of ProfileRelevST(TNG,P,StartTN,DestTN)), covering the circles of TripG(ProfileRelevST (TNG,P,StartTN,DestTN)) not being part of any direct connecting trips between (StartTN,DestTN), where each trip has at least one section in those circles not occurring as a circle section in

\footnotetext{
${ }^{9}$ Note that a direct connecting trip of TripG between (StartTN,DestTN) is not always a P-matching trip (if a preferred POI must be visited outside of it) but it is covered by a P-matching trip of the CCT with the minimum length possible.
} 
all others, and each trip has a minimum possible length with these conditions. ${ }^{10}$ A Maximal CLT (MCLT) is a CLT with the highest number of trips. The number MCLT (TNG, P, StartTN,DestTN) is the weighted count of trips in an MCLT, each multiplied by their MatchT PD value.

- Covering Spike-containing Trip set (CST), if it contains (non-direct) connecting or returning trips of ProfileRelevST(TNG,P, StartTN,DestTN)), covering the spikes (dangling sections) of TripG(ProfileRelevST (TNG,P,StartTN,DestTN)) not being part of any direct connecting trips between (StartTN,DestTN) or circles, where each trip has at least one section in those spikes not occurring as a section in spikes in all others, and each trip has a minimum possible length with these conditions. A Maximal CST (MCST)) is a CST with the highest number of trips. The number MCST (TNG,P,StartTN,DestTN), the number of is the weighted count of trips in an MCST, each multiplied by their MatchT PD value.

A union of a CCT and a CLT is called a Combined Covering C-L Trip set (CombCLT $)$, with its maximal size of MCombCLT $(T N G, P, \operatorname{StartTN}$, DestTN $)=$ $\operatorname{MCCT}(T N G, P, \operatorname{StartTN}, \operatorname{DestTN})+\operatorname{MCLT}(T N G, P, \operatorname{StartTN}, \operatorname{DestTN})$. A union of a CombCLT and a CST is called a Combined Covering Trip set (CombCT), with the size of a maximal one being MCombCT $(T N G, P, \operatorname{Start} T N, \operatorname{Dest} T N)=$ MCombCLT (TNG, P, StartTN,DestTN $)+M C S T(T N G, P$, Start TN,DestTN) - DupST where DupST is the number of trips occurring in both MCombCLT and MCST (having a back-and-forth section not covered by other trips of the covering set).

The summed novelty ratio of a trip sequence of an MCombCT in the order of increasing trip length is called the Maximal C-L-prioritized summed novelty ratio w.r.t. $(T N G, P, \operatorname{Start} T N, \operatorname{Dest} T N)$.

Example 5 The set of direct connecting trips between $D, X$ (in the graph without planned sections) are $\{(D, E, K, X),(D, M, Q, X),(D, M, Q, K, X),(D, E, K, Q, X)\}$. Either 3 of them forms a MCCT. Additionally, the singleton trip set $\{(D, E, K, L 1, L 2, K, X)\}$ is a $M C L T$, and an MCST is the trip set $\{(D, A, D, E, K, X),(D, E, B, E, K, X),(D, M, Q, N, Q$, $X),(D, E, K, X, Z, X),(D, M, Q, X, Z, W 1, Z, X),(D, E, K, X, Z, W 2, Z, X)\}$.

The following important statements follow from the respective definitions, due to the fact that if two different (shortest) trips with the same length share a section, the weight of that section will be the same if either one of the trips is selected for forming the index value; and the fact that if a section appears in multiple trips then its weight will be counted only with the(/a) shortest one for the index, and the first occurrence in a sequence for the summed novelty ratio.

Proposition 2 Trip variety (in terms of either index) of all possible trips for a profile (weighted by MatchTPD) equals to the trip variety (of the same index) of a maximal combined cover (for each of the variety indices).

Proposition 3 Trips being generated in the following (C-L-prioritized) order result in a combined covering trip set whose values of each trip variety indices are equal to that of

\footnotetext{
${ }^{10}$ Although the definition contains circles instead of loops, there is a matching with the loops (as each loop must contain a circle in our terms) and this way our current definition remains consistent with [12], and puts more emphasis on the loops as circle-containing returning trips or parts than the circles themselves.
} 
a maximal combined cover. First, connecting trips without loops and with the necessary spikes only (for preferred POI visit where needed), in the increasing order of their length, starting with the shortest one(s) possible. Second, trips with loops: returning trips or connecting trips containing at least one circle and with the necessary spikes only, in the increasing order of their length. Third, trips with spikes not having been covered by the previous trips, in the increasing order of their length. ${ }^{11}$

Proposition 4 The maximal C-L-prioritized summed novelty ratio is an upper bound for a possible summed trip novelty ratio for any sequence of ProfileRelevST (TNG, P, StartTN,Dest TN)) trips where each connecting trip precedes each returning trip, the first appearance of each non-returning-part-section of any possible connecting trip is not in a returning part of any trip, and the first appearance of each section of any circle in the network does not appear in a returning (back-and-forth) part of its first trip in the sequence. It is a strict bound if there is no partial P-matching trip (shorter than the range minimum) and no POI-preference given.

If a respective index equals to its covering number counterpart, it means all the respective trips / loops / spikes are independent (pairwise disjoint), so the network provides the maximal variety in that respect with the given number of (independent) opportunities. If a variety index is relatively small for a large covering number, it means there are quite a few trip / loop / spike part variations but with many overlaps.

Definition 12 Including the above defined counts, the Extended Composite Trip Variety Index is a 6-tuple (parameters omitted for the sake of simplicity): ExtCompTVI= (CTVI/MCCT,LTVI/MCLT,STVI/MCST), where the slash is used as a notational separator marker and does not denote division (however, treating it as a divider and counting the rations is also meaningful for measuring relative variety of the amount of trip opportunities provided, see later in Section 4).

Further properties of the indices are explored in the next section.

Next we consider the computation of the indices. Based on the last three propositions in the previous subsections, an algorithm is sketched for computing the combined trip variety index, by simulating generation of a proper (C-L prioritized) combined covering trip set, which might not be maximal, but assigns the same weights to sections as a maximal cover. The actual implementation may vary.

The following concepts are needed for the algorithm:

Definition 13 The POI-closeness of a trip (or a node) $x$ in a network TNG regarding a set of preferred POI types poiTypes $p$ specified by a profile $p$, denoted by PoiDist (TNG, x, poiTypes $)$, or if the context is obvious, in short PoiDist(x), is defined as:

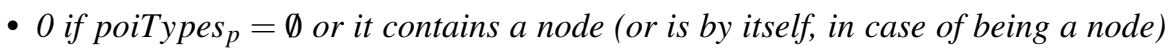
of a preferred POI type,

- the length of the shortest spike trip leading to a preferred POI if reachable in the allowed max range - minus the trip length of $x$ - given by $p$.

\footnotetext{
${ }^{11}$ In some cases, covering trips generated in the increasing order of their length does always not form a maximal cover, see, for instance, part a2 on Figure 2. But the variety index values will be the same as for a maximal cover.
} 
- $\infty$ if such POIs are not reachable using a spike trip having its length in the allowed range max given by $p$ (minus the trip length of $x$ ).

Note that the POI-closeness is twice the distance of the closest preferred POI of the respective trip or node.

Definition 14 The (POI-neutral) trip-distance of a node $n$ in a network TNG, denoted by TripDist $(T N G, n, P$, Start $T N$, Dest TN), regarding a profile $P$ (with the usual notations) and node sets (StartTN,DestTN) is the length of a shortest trip with the following conditions: it is either a connecting trip between (StartTN,DestTN) or a returning trip from StartTN $\cap$ Dest $T N$, visiting the node $n$ (no preferred POI visit is necessary). If this length is larger than max (tripRange $p_{p}$ ) it will be $\infty$. A similar definition can be given for sections $s$, for the shortest trip with the given condition including $s$.

The POI-visiting trip-distance of a node or section $x$, denoted by TripPoiDist(TNG, x, P, StartTN,DestTN), is similar to the above with the extra condition for the trips visiting a preferred POI if poiTypes $s_{p} \neq \emptyset$.

The (POI-visiting) Loop-length-distance of a loop l, denoted by LoopLengthPoiDist (TNG, l,P,StartTN,DestTN) is the length of the shortest trip with the following conditions: it is either a connecting trip between (StartTN,DestTN) or a returning trip from StartTN $\cap$ Dest $T N$, containing the loop $l$ and visiting a preferred POI if poiTypes $p \neq \emptyset$. If this length is larger than max(tripRange $p$ ) it will be $\infty$.

Proposition 5 Let TNG be a trail network graph with nodes $T N, P=\left(\right.$ actMod $_{p}$, tripType $_{p}$, tripRange $_{p}$, retOnly $_{p}$, accMod $_{p}$, poiTypes $\left._{p}\right)$ a trail user profile, Start $T N \subseteq$ $T N$ (possible starting locations) and the set of acceptable destination nodes Dest TN $\subseteq$ $T N$. Assume all elements of StartTN and DestTN are compatible with accMod . $_{\text {. }}$

The value of CompTVI $=(C T V I, L T V I, S T V I)$ for the above parameters can be computed with the following algorithm:

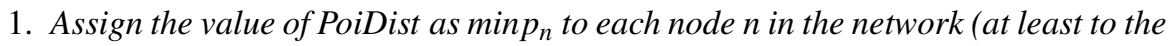
reachable nodes for $P$ - this can also be done dynamically during further steps).

2. Generate a cover of P-matching connecting trips CT Set in their increasing order

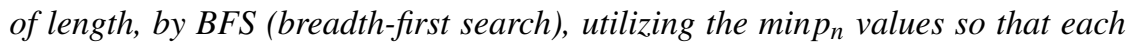
of these trips will be a direct connecting trip with an optional spike to the closest preferred POI. If Start $T N \cap$ Dest $T N \neq \emptyset$ than it must be separately done for each $a \in$ StartTN $\cap$ DestTN towards Dest $T N \backslash\{a\}$, so that no returning trips are included.

3. Assign to each non-spike section contained by trips of CTSet the length of the shortest trip of such (denoted by mint $D_{s}$ ). The value length $(s) /$ mint $_{\text {s }}$ will be the candidate for the eventual $w_{C T V I}$ of that section.

4. Assign the value of TripDist as mint ${ }_{n}$ and TripPoiDist as mint $p_{n}$ w.r.t. P to each node $n$ in the network (its reachable part for $P$ ) and similarly, mint , mint $_{\text {s }}$ for sections $s$ respectively. ${ }^{12}$

\footnotetext{
${ }^{12}$ This step can be done by generating an optimized cover of direct connecting trips DCTSet, regardless of POI visits, and (optional) spikes connected to these trips from each node. Note that in some cases, the two values mint $_{n}$ and mint $_{n}$ will be based on different trips, and neither of them must be based on a direct connecting trip in which node $n$ appears. Furthermore, if retOnly $y_{p}$ is true then this step must be done separately for each $a \in \operatorname{Start} T N \cap \operatorname{Dest} T N$, storing possibly multiple values for each $(a, n)$.
} 
5. Find and generate covering loops LT Set each having at least one section not yet scored by $w_{C T V I}$, in their increasing order of LoopLengthPoiDist, which is the value $\operatorname{mint}_{l}=\min \left(\left\{\operatorname{mint}_{k}+\right.\right.$ length $\left.(l) \mid k \in \operatorname{nodes}(l)\right\} \cup\left\{\right.$ mint $_{m}+$ length $(l)+$ min $_{n} \mid m, n \in$ nodes $\left.(l)\right\}$. This is going to be the length of the trip giving the weight value candidates for LTVI of the circle sections in the loop. Assign the value mint $L_{l}$ to each loop $l^{13}$

6. Assign to each circle section s contained by loops of LTSet the value of mint $L_{l}$ by a containing loop for which it is minimal. The value length $(s) /$ mint $_{l}$ will be the candidate for the eventual $w_{\text {LTVI }}$ of that section.

7. Remaining sections not covered yet by Steps 3 and 6 can only be parts of backand-forth sections (as parts of spikes or loops) of profile-relevant trips. Prune

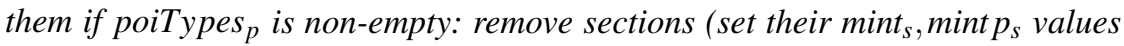
as $\infty)$ not being part of any loops of Step 6 or spikes leading to a preferred POI.

8. Assign to each remaining section having finite mint $p_{s}$ the value $\min S_{s}=$ $\min \left(\left\{\operatorname{mint}_{s}\right\} \cup\left\{\operatorname{mint} L_{l} \mid s \in \operatorname{sections}(l)\right\}\right)$. The value length $(s) / \operatorname{mint} S_{s}$ will be the candidate for the eventual $w_{S T V I}$ of that section.

9. Upscale the weights for short trips: if any value assigned to sections in Steps 3, 6,8 is lower than $\min \left(\right.$ tripRange $\left._{p}\right)$, then set it explicitly to to min(tripRange trip so that the weight will be the maximal possible value (c.f. MatchTPD).

10. Aggregate (sum by the respective trip variety index type CTVI,LTVI,STVI) and output the computed section weight values $w_{C T V I}, w_{L T V I}, w_{S T V I}$ for each relevant section as values of the combined trip variety index.

What the algorithm does is exactly how the indices are defined. There are three types of sections in the (reachable part) of the graph, getting their weights differently: A section of a direct connecting trip gets a CTVI weight, as the proportion to the length of the shortest profile-relevant connecting trip (including a preferred POI visit if necessary, by a spike). A Circle section of a trip containing a loop not being part of any direct connecting trip gets an LTVI weight, as the proportion to the length of the shortest profile-relevant trip with a loop containing it in a circle. A Back-and-forth section not being part of any of the above gets an STVI weight, as the proportion to the length of the shortest nondirect/returning trip (may be a non-circle part of a loop).

The algorithm is deterministic by counting the same weight for each section, and giving the same results, regardless of the order of the particular nodes and trips taken. It is guided by the length and trip format, and any variation beyond that yields the same weighting for each graph section (c.f. the reasoning for well-definedness of the indices).

\section{Examples and Discussion}

Example 6 Different simple trail network topologies (with no trip length restrictions or POI preferences) are shown on Figure 2 with their (approximate) values of ExtCompRTVI $=(L T V I / M C L T, S T V I / M C S T)$ (for returning cases) and

\footnotetext{
${ }^{13}$ Circles can be detected, for instance, by BFS, starting from each node being an endpoint of a section not covered yet by Step 3, or directly during the operation for Step 4, in parallel to assigning the values to nodes. Furthermore, if retOnly $y_{p}$ is true then this step must be done separately for each $a \in \operatorname{StartTN} \cap \operatorname{DestTN}$.
} 
a1)

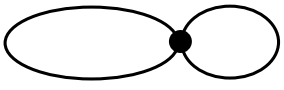

$(2 / 2,0)$

LTVI MCLT (no spikes)

d)

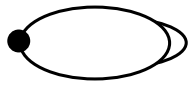

$(1.1 / 2,0)$ a2)

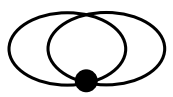

$(2 / 3,0)$

e)

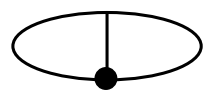

$(1.7 / 2,0)$ b)

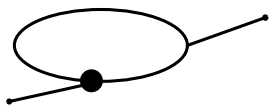

$(1 / 1,0.75$ / 2)

LTVI MCLT STVI MCST

f)

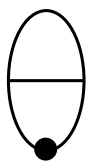

$(1.5 / 2,0)$ c)

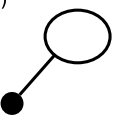

$(0.5 / 1,0.5 / 1)$

g)

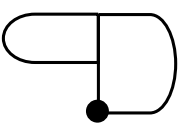

$(1.6 / 2,0)$

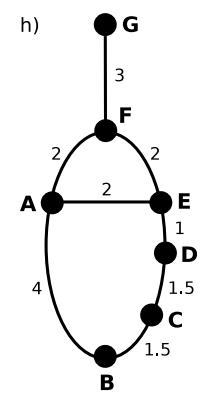

AEFA:

A: $A B C D E A$

$A F G<->$ :

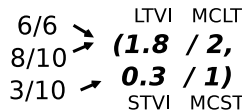

BCDEAB: $10 / 10$

B: $B C D E F A B: 4 / 12 \geq \mathbf{( 1 . 3 3 / 2}$

$B A F G<->: 3 / 18 \rightarrow 0.17 / 1)$

CDEABC: $10 / 10$

C: CDEFAEDC: $4 / 11 \geqslant(\mathbf{1 . 3 6} / \mathbf{2}$,

CDEFG<->: $3 / 15$ - 0.2 / 1)

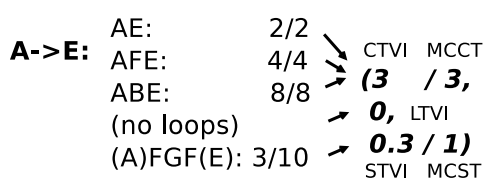

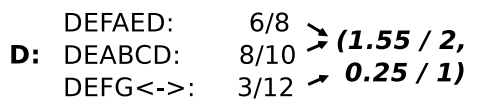

FAEF:

F: FABCDEF:

$6 / 6$

$8 / 12 \geq(1.67 / 2$,

$F G<->$ :

$3 / 6 \rightarrow 0.5 / 1)$

(G)FAEF(G) 6/12

G: GFABCDEFG: $8 / 18 \geqslant(0.94 / 2$,

$G F(A E F)<->: 3 / 12,0.25 / 1)$

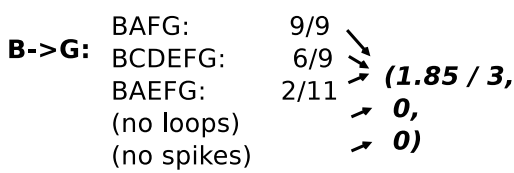

Figure 2. Sample cases with the Composite (Returning) Trip Variety Index, with two cases of connecting trips (extended variant of the figure in [12])

ExtCompTVI $=(C T V I / M C C T, L T V I / M C L T, S T V I / M C S T)$ (for the last two, connecting cases). ${ }^{14}$ We are referring these cases as illustrations for the properties listed below.

Proposition 6 The following general properties hold (and follow directly from their definitions), illustrated by the examples of Figure 2. They reveal more details of the meaning, the characteristics and benefits of these measures:

1. For returning trips, if the first (shortest, matching) loop trip is a circle, it gets a LTVI=1. Independent circle trips are counted as 1 each in the LTVI, therefore in this case, the LTVI is simply the number of them, and in general, it is a generalization of this number. Flower graphs show the maximal variety in loop trips (see case al). If there are independent circle trips, LTVI is least the number of them.

2. If all (direct) connecting trips are independent, CTVI equals the number of them, so it is a generalization of the number of independent direct connecting trips. CTVI is not lower than the numer of possible independent connecting trips.

\footnotetext{
${ }^{14}$ If any of them is 0 or not applicable for a case, we simply put a 0 instead of the formal $0 / 0$, which may have caused confusion. Recall that the / marker in the index tuples is a separator and not a division mark, although an alternative interpretation of it as a division gives meaningful, relative values.
} 
3. For returning trips, if the second shortest (matching) loop trip share 0.5 of its length with the first one, it gets the value of 0.5, and results a total of 1.5 in the LTVI (case f). If there is only a minor variant for a short section of a loop, its addition to the LTVI will be proportionally smaller (case d). If the two loops share only a shorter section, their LTVI will be near to 2 (cases $e$ and $g$ ).

4. The indices are invariant of the length of the actual trips (if they fall into the profile range), they only depend on the ratio of shared sections among them.

5. Each trail section is counted only once in the weighted lengths, in the shortest possible trip of the respective format (direct connecting, circle part, spike part) it is contained by. Namely, if a section is part of a direct connecting trip, it is added to the CTVI. Otherwise, if it is part of a circle, added to the LTVI. Sections used only in back-and-forth parts of trips are counted into the STVI (cases b and c).

6. If a circle (not part of a direct connecting trip) is cut (separated) at a point, its remaining sections will be counted into other circles they are part of, and the rest of the sections are transferred to STVI. After two independent spikes are joined to form a circle, their weights are transformed from STVI to LTVI.

7. Circles of P-shaped loops are downgraded proportionally to the distance of the circle from the starting/ending point (or shortest connecting trip). Their circle parts are valued by LTVI, and their back-and-forth parts by STVI. The more distant a circle is, the less relevant it is for LTVI (overdominated by closer sections).

8. Weights of sections constituting the index values may differ for different starting (or destination) nodes for the same profile, reflecting their local relevance.

9. Index values are continuous w.r.t. modifications in the network graph without topological changes (c.f. cases $d$ and $f$ ). A topological change may cause value transfers between components of composite indices.

10. If a section is removed by gradually decreasing its length and merging the two of its ending nodes, continuity remains if it does not eliminate or transfer a trip of a certain format relevant to the profile (direct connecting, trip with loop, trip without loop but spike). The same holds when spitting a node into two and gradually enlengthening it (c.f. cases al, $e$ and $g$, where two loop trips remain in each).

11. It follows from the above that if a node with a degree of 2 (a.k.a.pseudo-node) is eliminated by merging the two sections it connects (having the same properties), and the node is not a preferred POI, it will not influence any of the indices (except for the case of STVI for spike parts when no POI preference is given).

12. The STVI is however, sensitive to the number of (pseudo)nodes placed along a trip part (if no POI preference is given) as each possible turning point generates a different trip if it does not exceed the max length of the profile range. If this is not the intention, an adaptation of the STVI definition is necessary to treat only the longest possible spikes as separate trips.

13. If the length of a circle (not part of direct connecting trips) is gradually decreased to become 0, the LTVI gradually disappears and the spikes connected to it (reachable through it) will have a continuous change in the STVI.

14. If a spike section (a section not being part of any circle or direct connecting trip) is removed, it has no effect on the CTVI or LTVI. If a dangling loop (a loop part not being part of any direct connecting trip) is removed from the graph, it has no effect on the CTVI (if no preferred POI types are given).

15. The indices are not sensitive to hubs with multiple nodes close to each other, when the minimum trip length of the profile is significantly bigger than the distances inside the hub (the local loops and variations will cause an increase, although not significant, in the index). ${ }^{15}$

\footnotetext{
${ }^{15}$ An exception of this rule is when some circles starting and ending in such a hub actually do not return to
} 
16. the LTVI cannot distinguish between the cases of two independent loops of the same length, whether they are joined at their middle point or not (cases a1-a2). ${ }^{16}$ However, the MCLT will be different in the two cases (3 vs. 2). A similar effect can be observed when many but distant circles add up to 1 in the LTVI - their MCLT will be higher than 1, showing they do not form one single circular trip.

17. The 3 indices (CTVI,LTVI,STVI) have different meaning but they are additive and a summed index is also a characteristic measure (see the SumTVI definition).

18. After a user has taken at least MCLT number of different returning loop trips from the same, given node(/set), where each has at least one section not visited before, one must have been visited all sections of the network reachable from that node(s) by loop trips available for her/his profile The same is true for direct connected trips between (sets of) specific origin-destination nodes with MCCT.

19. For any (set of) node(s), there exists at least LTVI $+2 * S T V I$ number of returning trips, each of which, taken by a user in a sequence, having some section(s) not visited by the user before in that sequence.

20. The value of GTVI correspond to the maximal summed novelty ratio of possible series of subsequent matching trips taken by a user from a specific (set of) node(s) of the network (towards specific destination(s) and/or taking returning trips as specified by the profile and the start-destination node sets).

21. The actual values of the variety indices (in general, value SumTVI $=C T V I+L T V I+$ STVI) correspond the maximal summed novelty ratio of possible series of subsequent matching trips taken by a user from a specific (set of) node(s) of the network (towards specific destination(s)), with the $C$-L preference rule. ${ }^{17}$

22. A similar property as above holds for returning trips, with LTVI and STVI together, with the preference condition of each section being potentially part of a circle appears in a circle when it is first visited.

23. The ratio of CTVI/MCCT (divided) for direct connecting trips can be interpreted as a relative variety index, giving an average trip novelty ratio value for any sequence of a maximal CCT. In relaxed terms, if a user takes any covering series of (connecting) trips which have only the necessary (POI-visiting) spikes (a CCT, with each trip having at least one section not yet visited before), their average trip novelty ratio will not be lower than this value.

24. A similar property as above is true for loop trips with LTVI/MCLT for CLT, connecting trips with loop parts (with only the necessary, POI-visiting spikes) with (CTVI+ LTVI)/MCombCLT for CombCLT, and any covering set of trips with the conditions of Proposition 4 (CombCT) with $(C T V I+L T V I+S T V I) / M C o m b C T$ for CombCT.

the exact same node, but another one closer to it. In such a case, the (pseudo-)circle may be counted as part of a direct connecting trip (to CTVI), instead of a loop (to LTVI). To overcome this problem, the index must be improved by a minimum distance threshold for direct connecting trips (see future issues) or the network should be generalized before the indices are computed.

${ }^{16}$ Computation of the LTVI in such cases (as $a 2$ ) can be done in two different ways (either from the two independent loops as $1+1$, or starting with one of them and changing one of its parts with the other two trip part options in return as $1+1 / 2+1 / 2$ ), resulting the same value.

${ }^{17}$ More precisely, assume a user takes any series of profile-matching connecting trips between two (sets of) nodes in the network with the following restrictions: each section potentially being part of a direct connecting trip appears in a non-back-and-forth part of a connecting trip when it is first visited, and each section not directly part of any direct connecting trip but is potentially a section in a circle appears actually in a circle when it is first visited. Then, the following will be true for any section of these trips: the length of the section divided by the length of the first trip it appears in is not higher than the weight of the section as counted into either of the CTVI, LTVI,STVI indices. Summing these up for each trip, we can state simply that the novelty ratio of a trip in such a(n almost arbitrary) series of subsequent trips can not be higher than the actual sum of weights of its novel sections contributing to the $C T V I+L T V I+S T V I$ indices. 
Table 1. Trip variety computation: a detailed example of composite returning trip variety index calculation

\begin{tabular}{|c|c|c|c|}
\hline \multicolumn{4}{|c|}{ Variety of returning trips at node $\mathrm{N}$} \\
\hline \multicolumn{2}{|c|}{$\begin{array}{c}\text { Profile Pa } \\
5-20 \mathrm{~km} \text {, easy+difficult } \\
\text { nature+landscape preference }\end{array}$} & \multicolumn{2}{|c|}{$\begin{array}{c}\text { Profile } \mathrm{Pb} \\
\text { 3-10 km, easy only } \\
\text { no POI preference }\end{array}$} \\
\hline LTVI & STVI & LTVI & STVI \\
\hline \multicolumn{4}{|c|}{ Without planned sections } \\
\hline $\begin{array}{c}N Q K X Q N: \frac{6}{8} \\
N Q K L_{1} L_{2} K Q N: \frac{5}{11} \\
N Q M D E K Q N: \frac{8}{12}\end{array}$ & $\begin{array}{c}N Q(K) \rightleftharpoons \frac{1}{6} \\
(N Q) X Z \rightleftharpoons: \frac{2}{10} \\
(N Q K) E B \rightleftharpoons: \frac{1}{14}\end{array}$ & & $\begin{array}{c}{[N Q \rightleftharpoons]: \frac{1}{2} * \frac{2}{3}} \\
(N) Q X \rightleftharpoons: \frac{2}{6} \\
(N Q) X K \rightleftharpoons: \frac{2}{10} \\
(N Q) X Z \rightleftharpoons: \frac{2}{10}\end{array}$ \\
\hline$\sum$ TOTAL LTVI: 1.87 & $\sum$ TOTAL STVI: 0.44 & $\Sigma$ TOTAL LTVI: 0 & $\sum$ TOTAL STVI: 1.07 \\
\hline MCLT: 3 & MCST: 3 & MCLT: 0 & MCST: $3.67^{18}$ \\
\hline \multicolumn{4}{|c|}{ With planned sections (change effect) } \\
\hline$\ldots D B E \ldots:+\frac{3}{14}$ & $\operatorname{del}[E B \rightleftharpoons]:-\frac{1}{14}$ & $N Q K X Q N:+\frac{6}{8}$ & $\begin{array}{l}(N Q) K L_{1} \rightleftharpoons:+\frac{2}{10} \\
(N Q) K L_{2} \rightleftharpoons:+\frac{2}{10} \\
\operatorname{del}[Q X \rightleftharpoons]:-\frac{2}{6} \\
\operatorname{del}[X K \rightleftharpoons]:-\frac{2}{10}\end{array}$ \\
\hline$\sum$ TOTAL LTVI: 2.09 & $\sum$ TOTAL STVI: 0.37 & $\Sigma$ TOTAL LTVI: 0.75 & $\sum$ TOTAL STVI: 0.93 \\
\hline MCLT: 4 & MCST: 2 & MCLT: 1 & MCST: 3 \\
\hline
\end{tabular}

Intuitively, the value of the various trip variety indices is an idealized number of corresponding 'full' trips of a respective format (direct connecting, with loops, and the rest) a hiker could enjoy without repetitions for given start (and destination) nodes. The minimum number of actual exhaustive trips may be lower, because some sections belonging to multiple trips in a maximal cover can be combined into longer, more complex-shaped trips. The index is therefore not a minimum value, nor a maximum value of possible covering trips matching the given profile, but a value stating approximately how many independent trips the total 'novelty' will be equivalent to, if a user fully explores the profile-matching possibilities with any number of trips.

Referring back to our running example with the sample network graph, computations of the indices in different settings have been carried over, with the following results:

Example 7 Based on the trail network graph on Figure 1 and the profiles in Example 2, trip variety indices for the following nodes/sets are computed and analyzed, before and after the planned extensions (for each $G \in\{T N G, E x t T N G\}$ ), for each profile $P \in$ $\{P a, P b\}$. Results are shown on Figure 3. Sample computations are shown in Table 1.

1. Returning trips starting at node A (bus stop): $\operatorname{CompTVI}(G, P, A, A)$;

2. Returning trips starting at node $N$ (parking lot): $\operatorname{CompTVI}(G, P, N, N)$;

3. Returning trips starting at node $K$ (lodge): CompTVI $(G, P, K, K)$;

4. Connecting trips from A to $W 2$ (public transport): CompTVI $(G, P, A, W 2)$;

5. Connecting trips from nodes $W=\{W 1, W 2\}$ to nodes $L=\{L 1, L 22\}$ (from the city to the lake \& camp): CompTVI $(G, P, W, L)$.

Remarks below contribute to a better understanding of the meaning and behaviour of the trip variety indices, from an empirical point of view: 

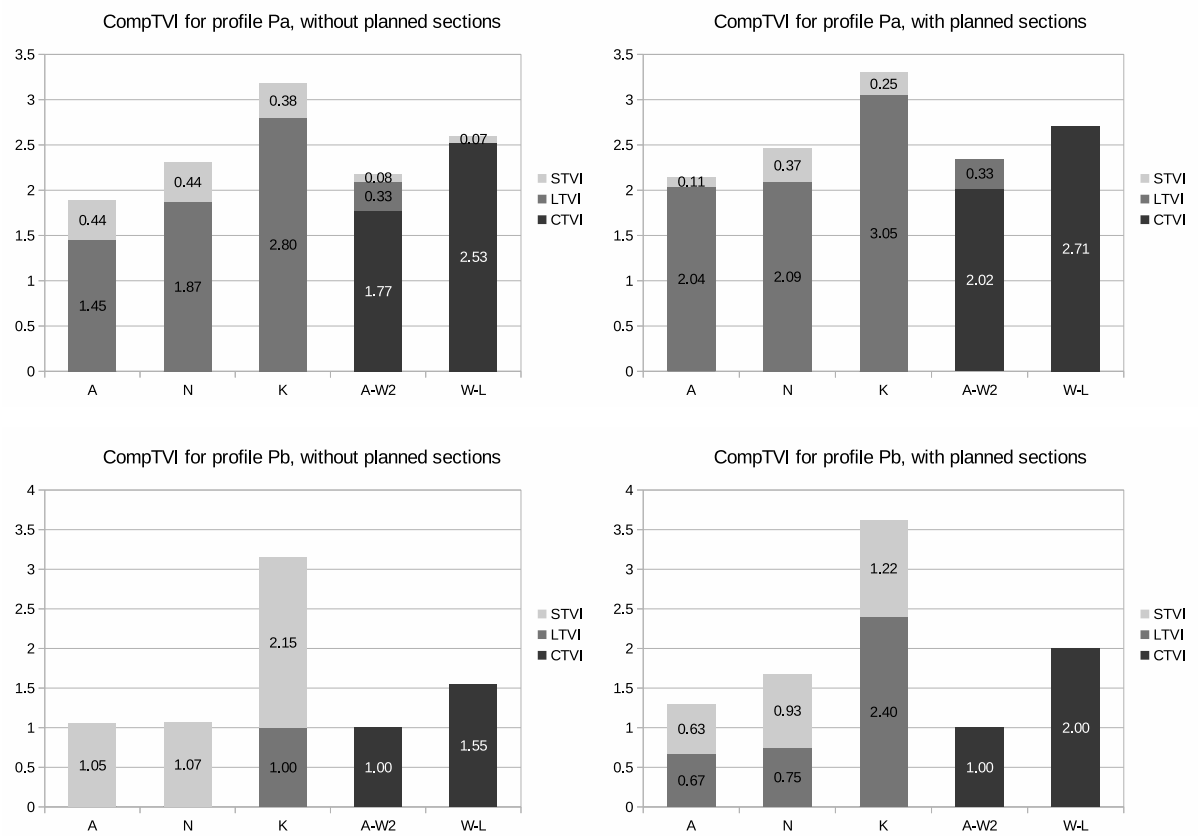

Figure 3. Trip variety index values for the network in Figure 1 with different profiles $P a$ and $P b$ of Example 2, for different nodes and trip formats (returning for $A, N, K$, connecting for $A \rightarrow W 2$ and $W=\{W 1, W 2\} \rightarrow L=\{L 1, L 2\})$, showing the impact of planned network improvements for each case

- As expected, implementing the planned sections in the network has a bigger impact on profile $P b$. While a $P b$-hiker was only able to take a loop trip from $K$ in the original network, the indices show a significant increase in the LTVI for the nodes $A, N, K$. The whole trail network became much more attractive for easy loop-trailfans. Moreover, the lake with nodes $L 1, L 2$ became directly reachable from $N$ for $P b$-hikers, without the need of an overnight stay at $K$. The $S T V I$ value remains relatively high for node $N$ even after loop trip opportunities came into the picture.

- At the same time, the planned sections have no impact on the variety of connecting trips for profile $P b$ at the observed node relations. This is because the direct connecting trips were already long for $P b$-hikers and no extra loops or longer variants became acceptable. An increase is, however, caused by the new improvements for profile $P a$, as all sections for the connecting trips can be incorporated into the direct parts of the trips or into a loop (to the lake, but that has not been changed).

- The difficulty upgrade of section $Q K$ has no effect on profile $P a$ since it has an effect on difficulty only, with the same length, so any change for profile $P a$ in the trip variety indices is caused by establishing the new section $D B$. The highest impact of it - as expected - can be observed at the returning trips from $A$, and a significant change is also caused at the connecting trips between $A$ and $W 2$. Interesting to see at the changes in varieties of other node relations for profile $\mathrm{Pa}$ that there are minor, but far-reaching impacts of this addition. The reason for this 
is the capacity of $P a$-hikers to walk long hikes, so that this far addition slightly increases the variety for them even between $W$ and $L$, for example. ${ }^{19}$

These trip variety indices are intended to model the trip variety and opportunities for users in recreational trail networks. So far, we have analyzed and observed some of their quantitative properties. Their initial variants - without their formal establishment yet - have been used in a real-life, pilot study case in [12] with promising results. For a thorough assessment and evaluation of their usefulness more experimental computations in real-life scenarios are needed. However, if we want to make sure we are on the right path, both with the definitions as they are introduced in this paper, and further, what kind of further evaluations and how they should be conducted, to see a broad view on the preferred ways of applicability of these, it is useful at this point to look at the modelbeing of these indices, in particular the composite CompTVI and its extended form. So we take a step back now, and look at it from a broader perspective and make a reflection about how and at what extent this can be taken as a model.

According to [18] a model is an instrument with the characteristics listed below. Although a detailed analysis is out of the scope of this paper, these aspects are briefly addressed in the following for the concept of the trip variety index:

Instrument for a purpose: recommendation and network management (change impact / design assessment). By having a sound definition and computation method, the indices can contribute to answering the question sets 1. \& 2., proposed in Section 1. A more specific utilisation portfolio shall be elaborated by looking at the properties and behaviour of the indices (see also sufficiency below).

Well-formedness: definitions and conventional notations introduced above ensure wellformedness of ExtCompTVI.

Adequacy: Compared to the simple combinatorial counting of the possible trips, or other conventional measures, the above presented properties support the adequacy of the index as being focused and purposeful. In terms of analogy, the measure itself is analogous to the centrality measures used in traditional graph theory and to the number of independent trips of respective formats. Furthermore, the index values (extended with the covering trip counts) show a correspondence between some intuitive min/max values for novelty ratios of series of trips the user may take, without representing the complexity of the trips themselves. Typical topology patterns and their variety indices have also been shown.

Justified: Index values are, by their definition, corroborated, coherent and conform, and falsifiable. Stability is achieved by several properties, including being continuous (small changes in the network, the from-to node sets or the profiles cause small changes in the index values), not sensitive to local hubs (with some exceptions), being independent of particular trip lengths, etc. Plasticity is achieved by the generality of the definition as being adaptable to any network topology, and the flexibility of the profile definition. Coherency and conformity can be improved for specific cases by proper adaptation of the definition(s), such as lifting the C-L preference condition for the price of the 3 indices not being additive any more.

\footnotetext{
${ }^{19}$ The paper [12] gives a real-life example when a 'winner' trailhead was identified with the most benefit (loop trip variety gain) of the changes in the network, while the institution initiated the changes was actually located at a different node of the network. For connecting trips, it has shown some far-reaching variety effects of local changes when relatively longer trips were allowed. Such an effect can be seen in our example as well.
} 
Sufficiency: To assess whether our indices are of firm quality and evaluated, such as of correctness, generality, usefulness, comprehensibility, parsimony, robustness, novelty, tolerance, modality, confidence and restrictions, specific experimental computations are needed for real-life networks, and verification with trail professionals. It is also required for assessing the possible acceptance by the community of practice. Other measures may supplement these indices to get a more profound insight, as our indices are based solely on the network structure and user profiles.

Grounding: There are surely non-explicit elements of grounding, but the network being modeled as a graph, or the trips as paths along the graph, the users as profiles with some preferences, etc. and the initial questions related to the purpose of the index (whether variety is a relevant measure and is related to the novelty ratio) can be identified as parts of the (indisputable) grounding.

Basis: The (disputable, adaptable) basis is formed by the particular way of our definitions. How a user profile looks like in particular, or whether the network is uni- or bidirectional, do direct connecting trips have priority over loops, do circles have priority over spikes etc. these are the adjustable parts of the background, but each adjustment needs a re-definition of the respective indices.

Context: It is a really important question in what network scenarios these indices are useful. Many areas of the world do not have such a complex trail network which would require these computations. However, there are countries and regions with complex networks having been emerged during the last century which provide many variations with a sometimes unclear or not easily comprehensible structure, partly managed by different stakeholders. In such contexts, we do believe these indices are useful for the given purposes. Local properties of paths, visitor counts, the level of service at facilities, personal relations to particular places are not considered, which may substantially modify the user preferences.

Utilization of the index needs more consideration. It can be viewed as a comparative value when different nodes or relations, or profiles are in question and the relative variety of the trip opportunities of the impact of a network change is to be assessed. It can also be viewed as a section-level measure, and a way of evaluating a section is to calculate some variety indices of the same network with the section included and without it.

Some current limitations and promising future directions are considered next.

Adapting the indices for directed graphs - as a possible future option - is closely related to the issue of giving, for instance, walking times based on elevation profiles instead of single distances as labels to the trail sections (c.f. [20,4]). This will lead to a more precise quantification, however, it is not sure such modification will worth the effort of redesigning the indices. The trip variety indices serve mainly as an approximate guide which can be refined according to other measures not represented here. The effects of these factors is likely to override the precision gained by a refined trip variety index.

Although the user profiles include preferences for POI types, the indices do not tell any information about the actual number of reachable POIs. The RPOI used in [12] seems to be a proper complementing measure.

The most important future work is to have more evaluations in real-life networks and experiment on how specific types of network change are reflected in the index values, and how these changes correlate to the changes in the index values to users' perceptions. As [7] presented a method for improving connectivity in urban cycle networks, these indices can be utilized in a similar manner with trail networks for outdoor recreation activities. 
Furthermore, a more profound analysis on user preferences regarding the types and properties of trip routes may reveal that different user profiles require different variety indices. Our composite trip variety index has a meta-directive as it prioritizes direct connecting trips over trips with loops or spikes, and loops over spikes.

\section{Conclusion}

In this paper, graph measures have been defined and formally established for the use in geographical information systems for modeling and quantifying the variety of user trip opportunities in - possibly complex - recreational trail networks (such as hiking paths or cycling ways), based on visitor profiles. Each index gives a score to either a single node, sets of nodes, a pair of nodes (origin-destination places), or a pair of sets of nodes reflecting on the variety of possible trips of specific formats around or between them. The indices can be used for ranking of these as potential locations of their activities - the larger the value of an index for a specific trip format is, the more variety is offered for possible trips by the network for specific user needs and preferences.

A formal establishment is given based on undirected graphs and simple trips (without repeating sections in the same direction), and a formal user profile model is proposed. Different forms of trip variety index values are can reflect on how different are the possible connecting trips between two nodes or node sets in the network, the possible loop trips or loop parts of connecting trips, or detour sections which can only be taken in a back-and-forth manner(spikes). Some of these indices were partially and informally introduced in [12], with a proposed agenda of formal definitions and assessment. This paper intends to serve that purpose, including the implementation-ready definitions of network, user profile and trip models as well as the algorithms of computing the indices.

The result is a solid, well-defined and theoretically verified index construction, with some initial computations on simple networks with promising results. A brief insight is also given on the model-being of these indices, revealing further properties and directives for future use and improvement. The main purpose or application area of the indices is the personal trip recommendation in complex systems (where to go for a greater variety of specific types of possible trips), and network change impact assessment (how does addition, deletion or upgrading a section effects on the various trip opportunities). This is a novel approach as being solely dependent on the network structure, similarly to graph centrality measures, enhanced with preferred nodes for visit (POI nodes).

Further experiments and computations in real-life trail networks must be taken in order to assess the comprehensibility and usefulness in the community of practice, as well as to develop effective visualisations and other methods of utilization. According to our knowledge, these indices reveal a new type of knowledge not having been commonly extracted and utilized from geospatial route network graphs in such a manner and for such purposes.

\section{Acknowledgement}

The research was supported by the Hungarian Ministry of Innovation and Technology NRDI Office within the framework of the Artificial Intelligence National Laboratory Program. 


\section{References}

[1] Wikiloc - trails of the world, https://www .wikiloc.com/, [Online; accessed 26-Jan-2021]

[2] OuterSpatial - a stewardship-first approach to data, maps and apps for recreation (2019), http: //www . alaska-trails.org/2019-trails-conference-presentations.html, [Acc. 25-Jan-2021]

[3] Brämer, R.: Profilstudie Wandern (2008), Deutsches Wanderinstitut

[4] Calbimonte, J.P., Martin, S., Calvaresi, D., Zappelaz, N., Cotting, A.: Semantic Data Models for Hiking Trail Difficulty Assessment, pp. 295-306 (01 2020)

[5] Cheng, Y.Y., Lee, R.K.W., Lim, E.P., Zhu, F.: Measuring Centralities for Transportation Networks Beyond Structures, pp. 23-39 (05 2015). https://doi.org/10.1007/978-3-319-19003-7_2

[6] Clark, R.N., Stankey, G.H.: The recreation opportunity spectrum: A framework for planning, management, and research. Tech. rep., Department of Agriculture, Forest Service, Pacific Northwest Forest and Range Experiment Station. (1979)

[7] Guillermo, L., Orozco, N., Battiston, F., Iñiguez, G., Szell, M.: Data-driven strategies for optimal bicycle network growth. Royal Society Open Science 7(12) (2020)

[8] Hong, J., Tamakloe, R., Lee, S., Park, D.: Exploring the topological characteristics of complex public transportation networks: Focus on variations in both single and integrated systems in the seoul metropolitan area. Sustainability 11(19) (2019), https : //www . mdpi . com/2071-1050/11/19/5404

[9] Lera, I., Pérez, T., Guerrero, C., Eguíluz, V.M., Juiz, C.: Analysing human mobility patterns of hiking activities through complex network theory. In: PloS one (2017)

[10] Márkus, Z., Wagner, B.: GUIDE@HAND: digital GPS based audio guide that brings the past to life. In: Pavlov, R., Stanchev, P. (eds.) Digital Preservation and Presentation of Cultural and Scientific Heritage. pp. 15-25. Bulgarian Academy of Sciences, Sofia (2011), http://eprints.sztaki.hu/6712/

[11] Molnár, A.J.: Trailsigner: A conceptual model of hiking trail networks with consistent signage planning and management. Information Modelling and Knowledge Bases XXXII (2021)

[12] Molnár, A.J.: Synergistic planning of long-distance and local trails: A twin case study of trail network development in Northern Transdanubia. Tourism Planning \& Development (2021). https://doi.org/10.1080/21568316.2021.1936148

[13] Natera Orozco, L.G., Deritei, D., Vancso, A., Vasarhelyi, O.: Quantifying life quality as walkability on urban networks: The case of budapest. In: Cherifi, H., Gaito, S., Mendes, J.F., Moro, E., Rocha, L.M. (eds.) Complex Networks and Their Applications VIII. pp. 905-918. Springer I.P., Cham (2020)

[14] Outdooractive: Outdooractive - Data Model, [Online; accessed 20-Jan-2020]

[15] Parent, C., Spaccapietra, S., Renso, C., Andrienko, G., Andrienko, N., Bogorny, V., Damiani, M.L., Gkoulalas-Divanis, A., Macedo, J., Pelekis, N., Theodoridis, Y., Yan, Z.: Semantic trajectories modeling and analysis. ACM Computing Surveys 45(4) (2013)

[16] Peterson, B., Brownlee, M., Marion, J.: Mapping the relationships between trail conditions and experiential elements of long-distance hiking. Landscape and Urban Planning 180 (2018)

[17] Semenov, A., Zelentsov, V., Pimanov, I.: Application suggesting attractive walking routes for pedestrians using an example of saint-petersburg city. Procedia Computer Science 156, 319-326 (2019), 8th International Young Scientists Conference on Comp. Sci., YSC2019, 24-28 June 2019, Heraklion, Greece

[18] Thalheim, B., Nissen, I., Allert, H., Berghammer, R., Blättler, C., Börm, S., Brückner, J.P., Bruss, G., Burkard, T., Feja, S., Hinz, M., Höher, P., Illenseer, T., Kopp, A., Kretschmer, J., Latif, M., Lattmann, C., Leibrich, J., Mayerle, R., Wolkenhauer, O.: Wissenschaft und Kunst der Modellierung (Science and Art of Modelling), Philosophical Analysis, vol. 64. De Gruyter (05 2015)

[19] Vias, J., Rolland, J., Gómez, M., Ocaña, C., Luque, A.: Recommendation system to determine suitable and viable hiking routes: a prototype application in sierra de las nieves nature reserve (southern spain). Journal of Geographical Systems 20 (2018)

[20] Witt, P.J.: The development of a predictive hiking travel time model accounting for terrain variations. In: T. Jekel, A. Car, J. Strobl, \& G. Griesebner (Eds.), GI_Forum 2012: Geovisualization, Society and Learning. pp. 102-112. Salzburg (2012) 